\title{
September 2017 - January 2018
}

\section{September}

MobileHCl '17: 19th International Conference on Human-Computer Interaction with Mobile Devices and Services (Vienna, Austria)

Conference Dates: September 4-7, 2017

$\rightarrow$ http://mobilehci.acm.org/2017/

Ubicomp 17: ACM International Joint Conference on

Pervasive and Ubiquitous Computing

(Maui, Hawaii, USA)

Conference Dates:

September 11-15, 2017

$\rightarrow$ http://ubicomp.org/ubicomp2017

AutomotiveUI '17: 9th International Conference on Automotive User Interfaces and Interactive Vehicular Applications (Oldenberg, Germany) Conference Dates:

September 24-27, 2017

$\rightarrow$ http://www.auto-ui.org/17/

INTERACT '17: 16th IFIP TC 13 International Conference on Human-Computer Interaction (Mumbai, India)

Conference Dates:

September 25-29, 2017

$\rightarrow$ http://interact2017.org

\section{October}

CHIPLAY '17: Annual Symposium on Computer-Human Interaction in Play (Amsterdam, Netherlands)

Conference Dates: October 15-18, 2017

$\rightarrow$ http://chiplay.acm.org

SUI 17: Symposium on Spatial User Interaction (Brighton, UK)

Conference Dates: October 16-17, 2017

$\rightarrow$ http://www.sui2017.org

ISS 17: Interactive Surfaces and Spaces (formerly ITS)(Brighton, UK) Conference Dates: October 17-20, 2017 $\rightarrow$ http://iss.acm.org/2017

UIST '17: 30th Annual ACM Symposium on User Interface Software and Technology (Quebec, Canada)

Conference Dates: October 22-25, 2017

$\rightarrow$ http://uist.acm.org

\section{November}

VRST '17: 23rd ACM Symposium on Virtual Reality Software and Technology (Gothenburg, Sweden)

Conference Dates: November 8-10, 2017 $\rightarrow$ http://vrst.acm.org/vrst2017/

ICMI 17: International Conference on Multimodal Interaction (Glasgow, UK) Conference Dates: November 13-17, 2017 $\rightarrow$ https://icmi.acm.org/2017/
ACI 17: Fourth International Conference on Animal-Computer Interactions (Milton Keynes, UK) Conference Dates: November 21-23, 2017

$\rightarrow$ http://www.aci2017.org

MUM 17: International Conference on Mobile and Ubiquitous Multimedia (Stuttgart, Germany)

Conference Dates:

November 26-29, 2017

$\rightarrow$ http://www.mum-conf.org/2017/

OzCHI 17: Annual Meeting of the Australian Special Interest Group for Computer Human Interaction (Brisbane, Australia) Conference Dates:

November 28-December 1, 2017

$\rightarrow$ http://www.ozchi.org/2017/

\section{January}

Group '18: ACM Conference on Supporting Groupwork (Sanibel Island, Florida, USA)

Conference Dates: January 7-10, 2018

$\rightarrow$ http://group.acm.org/conferences/ group18/ 